\title{
Robust Accurate Extrinsic Calibration of Static Non-overlapping Cameras
}

Andreas Robinson, Mikael Persson and Michael Felsberg

The self-archived postprint version of this publication is available at Linköping University Institutional Repository (DiVA):

http://urn.kb.se/resolve?urn=urn:nbn:se:liu:diva-145371

N.B.: When citing this work, cite the original publication.

The original publication is available at www.springerlink.com:

Robinson, A., Persson, M., Felsberg, M., (2017), Robust Accurate Extrinsic

Calibration of Static Non-overlapping Cameras, Computer Analysis of Images and Patterns, 342-353. https://doi.org/10.1007/978-3-319-64698-5_29

Original publication available at:

https://doi.org/10.1007/978-3-319-64698-5 29

Copyright: Springer Verlag (Germany)

http://www.springerlink.com/?MUD=MP 


\title{
Robust Accurate Extrinsic Calibration of Static Non-overlapping Cameras
}

\author{
Andreas Robinson, Mikael Persson, and Michael Felsberg \\ Linköping University, 58183 Linköping, Sweden \\ firstname. lastname@liu.se, \\ https://liu.se/organisation/liu/isy/cvl
}

\begin{abstract}
An increasing number of robots and autonomous vehicles are equipped with multiple cameras to achieve surround-view sensing. The estimation of their relative poses, also known as extrinsic parameter calibration, is a challenging problem, particularly in the non-overlapping case. We present a simple and novel extrinsic calibration method based on standard components that performs favorably to existing approaches. We further propose a framework for predicting the performance of different calibration configurations and intuitive error metrics. This makes selecting a good camera configuration straightforward. We evaluate on rendered synthetic images and show good results as measured by angular and absolute pose differences, as well as the reprojection error distributions.
\end{abstract}

\section{Introduction}

Autonomous vehicles and robots benefit greatly from visual surround view sensing for both spatial and semantic understanding [15]. Surround view in turn requires multiple cameras with highly accurate extrinsics. Achieving the latter is an issue in camera configurations with limited or non-overlapping fields-ofview, as the standard methods for overlapping cameras cannot be applied. Since calibration errors cause biased estimates in crucial tasks such as visual odometry and spatial reconstruction, highly accurate estimates are required. Unfortunately non-overlapping configurations are common due to monetary and computational cost considerations and existing applicable methods rely on complicated tools and software.

Two approaches to minimal camera surround view sensing exist: either maximize the field-of-view per camera, with fisheye lenses or a catadioptric mirror, or allow more cameras with narrower field-of-view but rectilinear wide-angle lenses. While both fisheye lenses and catadioptric mirrors have excellent field-of-view, they can be costly and suffer from distortion that is difficult to calibrate well, compared to rectilinear lenses. In addition, with very few cameras it is hard avoid the body of the vehicle obstructing the view. Hence, this work will focus on the rectilinear type.

The intrinsic parameters of each lens in a camera configuration is straightforward to estimate independently [20]. We address the extrinsic estimation prob- 
lem, under the assumption that intrinsic calibration has been achieved to sufficient accuracy.
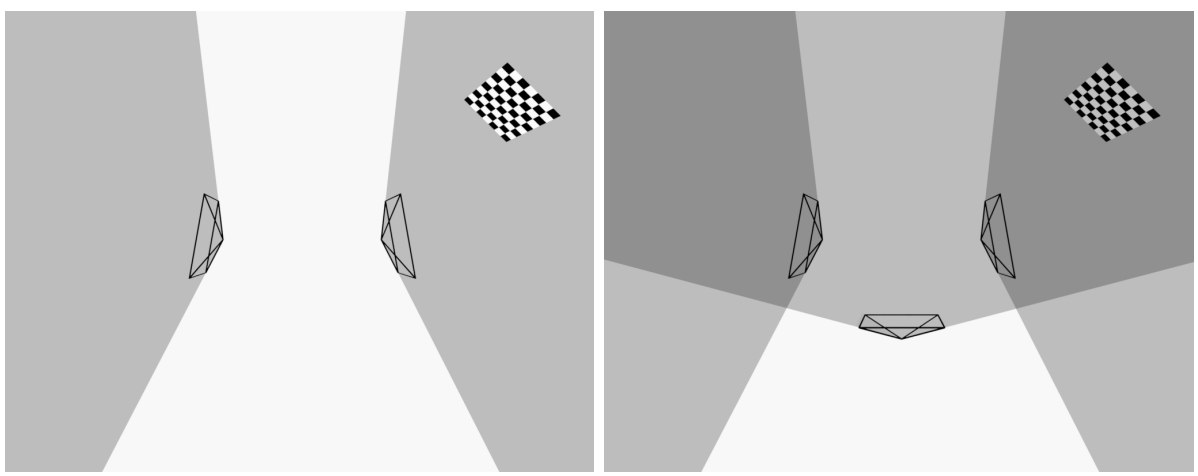

Fig. 1. Left: Camera pair without overlap seen from above. Extrinsic calibration not possible in a static setting. Right: A temporarily placed additional camera enables extrinsic calibration.

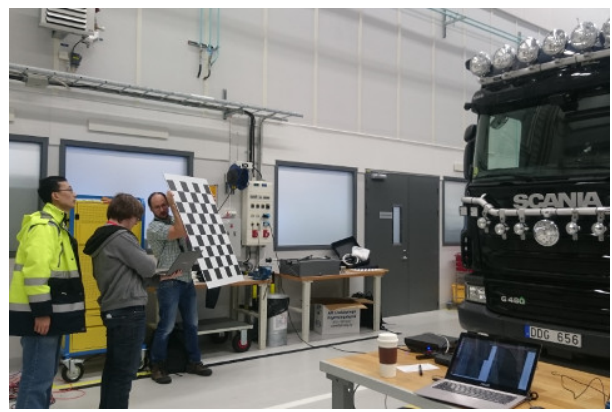

Fig. 2. Calibrating two marginally overlapping cameras on a truck, with the addition of a third camera.

We propose adding intermediate cameras during the extrinsic calibration process, as exemplified by the schematic in figure 1, for the purpose of calibrating cameras on vehicles such as the one shown in figure 2. This strategy creates or increases the overlap between fields-of-view and allows the use of any standard stereo calibration toolbox, with minimal development effort. Since the extra cameras are temporary, the cost, weight and performance limitations of the permanently installed cameras do not apply. Thus, better-performing cameras can be used to further improve the accuracy, if required. We show that greater 
overlap is beneficial and analyze the trade-off between additional cameras and propagated calibration errors.

In this paper we contribute

- a simple extrinsic calibration procedure based on applying the standard method through the novel use of additional temporary intermediate cameras.

- an evaluation framework for determining the expected quality of a given setup. This framework can also be used to identify good calibration configurations.

- a geometrically intuitive extrinsic error metric - the reprojection error distribution caused by the extrinsic error in a given camera setup.

\section{Related Work}

Approaches to calibrating non-overlapping cameras can be sorted into two broad categories, static and dynamic: Static methods either use mirrors to allow all cameras to see the same calibration object or large calibration objects that are only partially visible in each camera. Dynamic methods use structure-frommotion to capture the structure of a scene and to subsequently infer rigid relationships between cameras.

In the first category, a fairly recent method by Sturm et al. [18] recovers the poses of virtual cameras reflected in three or more planar mirrors, in order to find the pose of a "true" camera. Later work by several other authors $[10,9,11,17,19$, 14] present extensions and alternative approaches that also use planar mirrors. In contrast, Agrawal et al. [1] show that calibration with a spherical mirror is advantageous; it lacks degeneracies and only one mirror is required, although the accuracy is comparably low. Additionally, methods using calibration objects that are only partially visible in each camera have been suggested although this seems less common. Liu et al. [13] performs extrinsic calibration with a long stick, with the ends visible in each of the two cameras. In [12], this stick is substituted by lasers.

In the second category, calibration with structure-from-motion or photogrammetry, Esquivel et al. [6] and Carrera et al. [4] capture sequences of the surrounding environment on vehicles with rigidly coupled cameras, finding the relative camera poses after 3D reconstruction. Pagel et al. [16] implements online calibration based on a system for visual odometry and more recently Dong et al. [5] places easily identifiable calibration objects in a scene, uses photogrammetry to pinpoint their locations and then relate the cameras to be calibrated to these objects. Note that structure-from-motion approaches require additional information to determine an absolute scale. This is typically achieved with a calibration object of known size.

There are several ways to verify and compare camera intrinsic/extrinsic calibration quality. One choice is to verify that the reprojection errors on the calibration features is sensible, i.e. less than one pixel on average with no severe outliers. 
Another reasonable metric for comparison is the difference between the true and the estimated pose, as they map to intuitive physical properties. Since our target application is structure-from-motion, we propose that the reprojection error distribution provides an intuitive understanding of both error severity and acceptable limits.

Both static and dynamic methods can yield good results but have their downsides. Mirrors must have the reflective surface on the front to avoid chromatic aberrations, which adds to their fragility. Placing them to ensure the calibration object is reflected into the camera can be complicated. Large calibration objects warp under their own weight, while lasers have little pixel coverage and are hard to spot in cameras equipped with wide-angle lenses. Structure-from-motion approaches can be labor intensive to execute well in uncontrolled environments. We have also found that the estimated metric scale is very sensitive to noise. In addition, the perhaps most important aspect of these methods is that they require significant software development efforts to get up and running.

In contrast, with our method a few additional cameras makes the stereo calibration tools in the OpenCV software library [3] sufficient. The performance of the proposed hardware extension based approach has to our knowledge not been investigated previously.

\section{Method}

This section presents a general model of the extrinsic camera calibration problem, the proposed method, the imaging model and concludes with the error metrics.

\subsection{The Extrinsic Graph}

Consider the extrinsic camera configuration problem for $N$ cameras as an acyclic, un-weighted and un-directed graph. Each node corresponds to a camera pose and each edge to an observed relative pose. A connected graph implies that all poses are constrained and can be determined. Since the graph is acyclic, the relative poses can be found separately per constraint. General camera configurations need not result in a connected graph, but by adding cameras we can always create one. Each extra camera adds two new edges to the graph. Since acyclic connected graphs are sufficient, there is no need to add any cycle. Adding cycles and appropriate weights could improve accuracy, but would require joint optimization.

\subsection{Calibration Procedure}

Given the relative poses, any camera can be selected as origin and the poses of each other camera in relation to it are trivially computed by chaining the transformations. We use unit quaternion based poses to avoid compounding numerical errors. Thus we create an acyclic connected graph and estimate the relative poses in an interleaved manner as follows: 
Estimating Relative Transforms The metric relative transform between two overlapping cameras with known intrinsics can be estimated using a chessboard pattern of known proportions and a small number, (here $N=5$ ), of images. The chessboard pattern is found by OpenCV findChessBoardCorners() and the relative pose is computed using stereoCalibrate() which implements a homography based solver. Note that the signal-to-noise ratio of the estimation is related to the detection noise over the pixel distance between corner projections [8]. This suggests it is a good idea to have a large overlap between cameras and for the chessboard to cover most of it. Thus we require a lower bound of overlap to consider two cameras connected, how the overlap relates to the error is explored in section 4 .

Adding Cameras The goal is to create a fully connected acyclic graph by adding cameras. Finding the optimal configuration is both difficult and unnecessary. This motivates the following simple greedy scheme:

Iterate until the graph is connected:

- Find the two closest, in terms of angular distance, unconnected, cameras.

- Place an evenly distributed sequence of cameras with sufficient overlap along the shortest path.

The suitable number of cameras can be found by simulation similar to the one in section 4 . Note that regardless of the number of intermediate camera positions, at most two extra physical cameras are required since they can be moved.

\subsection{Camera Noise Model}

The image capture and feature extraction processes contain three distinct sources of noise:

In the optical stage, light enters the camera and is focused by the lens before hitting the sensor. Imperfections due to both lens design and manufacturing variability, attenuates (vignetting), diffuses (blurring) and distorts the light. We model the first two effects directly as

$$
I_{1}(\mathbf{x})=\cos ^{4}\left(k_{1}|\mathbf{x}|\right) \circ\left(H_{\sigma} * I_{0}\right)(\mathbf{x})
$$

where $|\mathbf{x}|$ is the distance from the camera's optical axis, $H_{\sigma}(\mathbf{x})$ is a small point spread (blur) Gaussian function of width $\sigma$, and $k_{1}$ controls the falloff rate of the vignetting function. In addition $(\cdot \circ \cdot)$ and $(\cdot * \cdot)$ denote point-wise multiplication and convolution, respectively. In the experiments, the blur kernel is fixed to $5 \mathrm{x}$ 5 pixels, with $\sigma=0.5 . k_{1}$ is chosen so that the maximum intensity at the left and right image edges are $25 \%$ of the maximum in the center. The third effect, distortion, is modeled below.

In the electronic stage, light is converted into electric charge in the sensor, is read out, amplified and digitized to form a digital image. Here, a significant 
source of error is the shot noise. We model this with a Poisson process approximation, specifically Gaussian noise with variance proportional to the light intensity:

$$
I_{2}(\mathbf{x})=\mathcal{N}\left(I_{1}(\mathbf{x}), b+k_{2} \sqrt{I_{1}(\mathbf{x})}\right)
$$

where $b$ is the minimum noise variance and $k_{2}$ a scale factor. We found that with $I(x) \in[0,255]$, setting the parameters to $b=2$ and $k_{2}=0.1$ produced noise that looked "reasonably realistic" as is shown in figure 4 . Estimating the parameters of a specific camera sensor would further improve the model accuracy.

In addition, pixel intensities are clamped to the range $[0,255]$, i.e

$$
I_{3}(\mathbf{x})=\min \left(\max \left(I_{2}(\mathbf{x}), 0\right), 255\right)
$$

In the digital stage, a detector locates the calibration pattern landmarks with subpixel-precision. This operation does not have to be modeled explicitly since either the OpenCV function findChessboardCorners () or cornerSubPix () can be applied directly. The output from the detector is a set of $2 \mathrm{D}$ points $\mathbf{y}_{k}$. $k \in[1, K]$ where $K$ is the number of calibration pattern landmarks.

In a real-world setting, these coordinates will have rather large errors due to lens distortion. However, for the purpose of the simulation we assume that distortion is mostly corrected by a function $U(\mathbf{y})$ and model the residual error with a Gaussian noise process. The final coordinates are thus

$$
\mathbf{z}_{k}=U\left(\mathbf{y}_{k}\right)+\epsilon_{d}
$$

with $\epsilon_{d} \sim \mathcal{N}\left(\mathbf{0}, \sigma_{d} \mathbf{I}\right)$, where $\mathbf{I}$ denotes the $2 \times 2$ identity matrix.

\subsection{Error metrics}

As mentioned in section 2, we consider two quality measures: the pose (rotational and translational) error and the reprojection error distribution. The latter provides a intuitive understanding of what impact the former is expected to have, on a structure-from-motion system.

Rotation and Translation Errors Let the $4 \times 4$ matrix $\mathbf{P}$ represent a $3 \mathrm{D}$ rigid transform, composed of a rotation matrix $\mathbf{R}$ and translation vector $\mathbf{t}$. Let $\mathbf{P}$ denote the ground truth and $\hat{\mathbf{P}}$ the associated estimate. $\mathbf{P}_{i}$ is the transform from world coordinates to the local coordinate system of camera $i$ and $\mathbf{P}_{j i}$ is the relative transform from the local coordinate system of camera $i$ to that of camera $j$.

We define the translation error of $\hat{\mathbf{P}}$ relative to $\mathbf{P}$ as $\epsilon_{t}=\left\|\hat{\mathbf{R}}^{-1} \hat{\mathbf{t}}-\mathbf{R}^{-1} \mathbf{t}\right\|_{2}$. Similarly, the rotation error is defined as $\epsilon_{R}=\left|\mathbf{R} \hat{\mathbf{R}}^{-1}\right|$, where $|\cdot|$ is the magnitude of the angle around the axis of rotation in $\mathbf{R} \hat{\mathbf{R}}^{-1}$. 
Expected Reprojection Error The distribution of reprojection errors is approximated by sampling points $\mathbf{y}$ and computing the error as follows. Let $\mathbf{y}=$ $(x, y, z, 1)^{T}$ be a homogeneous 3D point in the local coordinate system of camera $i$ and that $\mathbf{x}=\mathbf{P}_{i}^{-1} \mathbf{y}$ is the same point in the world coordinate system. With the camera projection operator defined as Proj : $(x, y, z, 1)^{T} \mapsto(x / z, y / z, 1)^{T}$, the reprojection error (in pixels) of $\mathbf{x}$ in camera $i$ is $\epsilon_{p}=f\left\|\operatorname{Proj}\left(\hat{\mathbf{P}}_{i} \mathbf{x}\right)-\operatorname{Proj}\left(\mathbf{P}_{i} \mathbf{x}\right)\right\|_{2}$, where $f$ is the focal length.

\section{Experiments}

The following experiments on synthetic imagery will show how the calibration error as measured by our chosen metrics varies with the camera configuration and the noise. Care has been taken to simulate realistic noise, as simpler models may hide interesting behaviors.

Our use-case, shown in figure 2, is a mobile platform (truck) with two 1600 x 1200 pixel cameras at 90 degree relative yaw angle but with very wide-angle lenses (focal length $1.67 \mathrm{~mm}$ ). Due to the wide fields-of-view, there is an overlap of approximately 10 degrees between the cameras. However, this image area is small and calibration patterns must be placed a significant distance away to be simultaneously visible in both cameras. The procedure has been performed on the mobile setup but the accuracy cannot be determined without ground truth or additional sensors. Thus, the experiments are performed on synthetic data.

\subsection{Setup}

To evaluate the impact of additional cameras providing significant overlap, a synthetic calibration pipeline is constructed. The pipeline generates noise-free images, applies noise, and finds the extrinsic camera parameters. We experiment with two cameras with a 90 degree interval, three cameras at 45 degrees, and five cameras at 22.5 degrees.

First, images are generated with the Blender 3D modeling software [7]. Pairwise camera configurations and associated pattern placements are shown in figure 3 . The cameras are outward facing on a circle with 1.5 meter radius and relative rotations of either $22.5,45$, or 90 degrees. In each case, five checkerboard calibration patterns (7-by-9 squares, $84 \times 108 \mathrm{~cm}$ ) are manually placed to maximally cover the joint field of view. As is shown in the figure, larger relative rotations provide less overlap and require the patterns to be placed farther away from the cameras. Note that cases such as 45-to-90 degree or 45-to-67.5 degree rotations are omitted, as they are equivalent to simultaneously rotating all objects in any of the existing setups.

In each configuration one calibration pattern at a time is placed inside the joint field-of-view of the camera pair. Images are rendered and the associated ground-truth image 2D coordinates of all pattern landmarks are stored.

Second, errors caused by the camera are simulated and added to the images. An example image with blur, vignetting and shot noise applied, is shown in figure 4 . 

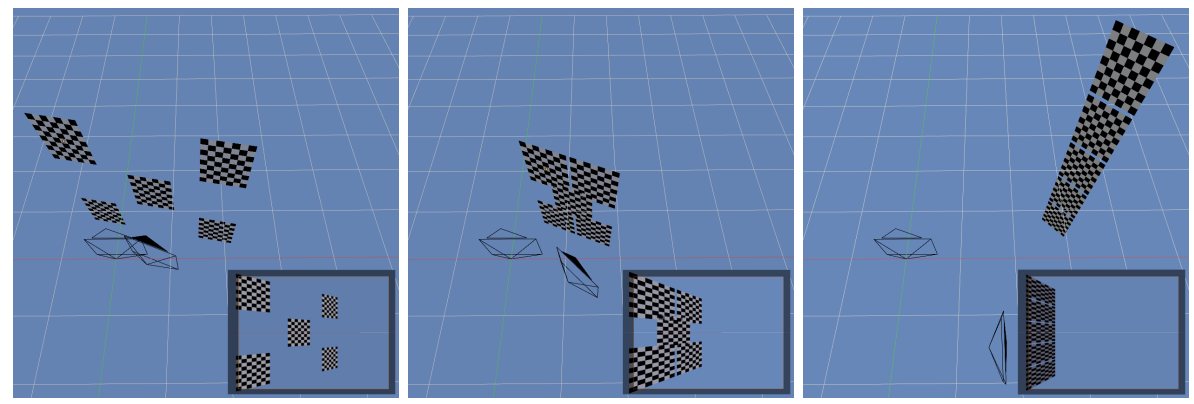

Fig. 3. Camera and pattern setups with 22.5 , 45 and 90 degree rotations between cameras. Insets show the pattern placements in the viewport of the right camera in the pair. Their appearance in the left camera are mirror-symmetric. The ground-plane grid lines are 1 meter apart.
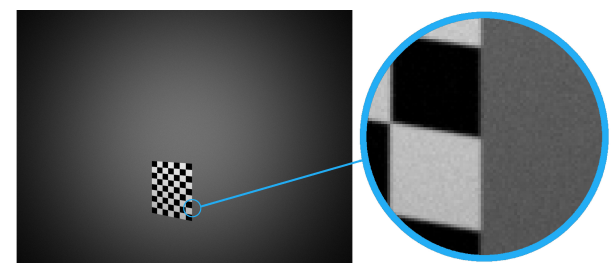

Fig. 4. Example of a rendered image with added vignetting, blur and shot noise. Inset: Closeup of camera shot noise - best viewed on-screen.

Third, the calibration pattern landmarks are located with sub-pixel precision (findChessboardCorners () in OpenCV). Since a coarse alignment can and should be verified manually, we only perform the subpixel refinement (cornerSubPix ()) with ground-truth landmarks as input. Gaussian noise is then added, simulating the effects of the residual camera distortion error. This is in line with other work, such as [1] and is in effect the main source of noise in the calibration pipeline.

Finally, these noisy landmark coordinates are used to estimate the relative transform between the two cameras in a pair (stereoCalibrate()).

For each of the camera configurations, we generate $M=1000$ calibration runs, with different noise added each time. This provides a reasonable sampling of the error distribution. N-view camera setups are simulated by linking the relative poses of pairs of cameras $\hat{\mathbf{P}}_{N, 1}=\prod_{k=1}^{N-1} \hat{\mathbf{P}}_{k+1, k}$. For example, in a threeview setup, $\hat{\mathbf{P}}_{31}=\hat{\mathbf{P}}_{32} \hat{\mathbf{P}}_{21}$. In addition we compute the reprojection errors of 10,000 random points $\mathbf{y}=(x, y, z, 1)^{T}$, uniformly distributed across the image plane at uniformly random depths $z \in[0.1,100]$. 


\subsection{Results}

The mean errors $\epsilon_{R}$ and $\epsilon_{t}$ in $\hat{\mathbf{P}}_{N, 1}$ are shown in figure 5. The distribution of reprojection errors in the presence of varying levels $\sigma_{d}$ of distortion noise $\epsilon_{d}$, are shown in figure 6 .
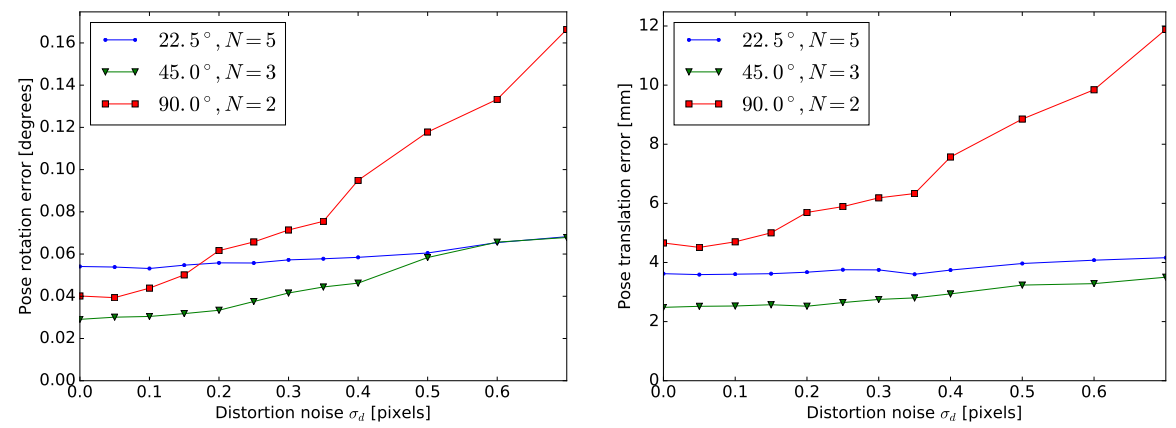

Fig. 5. Expected pose rotation errors and expected pose translation errors as functions of added $\epsilon_{d}$ noise of magnitude $\sigma_{d}$.

\section{Discussion}

It is clear from figure 5 that the calibration is most sensitive to noise when no cameras have been added $(N=2)$ and the overlap is small. In contrast, additional cameras $(N=3$ or $N=5)$ and greater overlap significantly improves the resilience to noise and is likely to compensate for the error accumulation along the chain of transforms. The difference between $N=3$ and $N=5$ is small. Depending on the assumed error, it is better to use one rather than three additional cameras. This trade-off may change if we expect significantly higher noise but that could also indicate poor intrinsic estimation quality. Presumably this generalizes to similar configurations, but should be confirmed using the provided toolbox to analyze the configuration on a case by case basis.

While it is difficult to compare the expected accuracy under the radically different setups and scenarios, we compare our results with those from other papers in table 1 . When possible, the values corresponding to a noise level of $\sigma_{d}=0.5$ are used.

Subject to differences in noise models, setup, cameras, and evaluation, we conclude that the performance of the proposed system is favorable or at least comparable to the baselines. Note that the rotation error represents a lower bound on projection accuracy since it will affect distant points which are otherwise unaffected by translation error. 

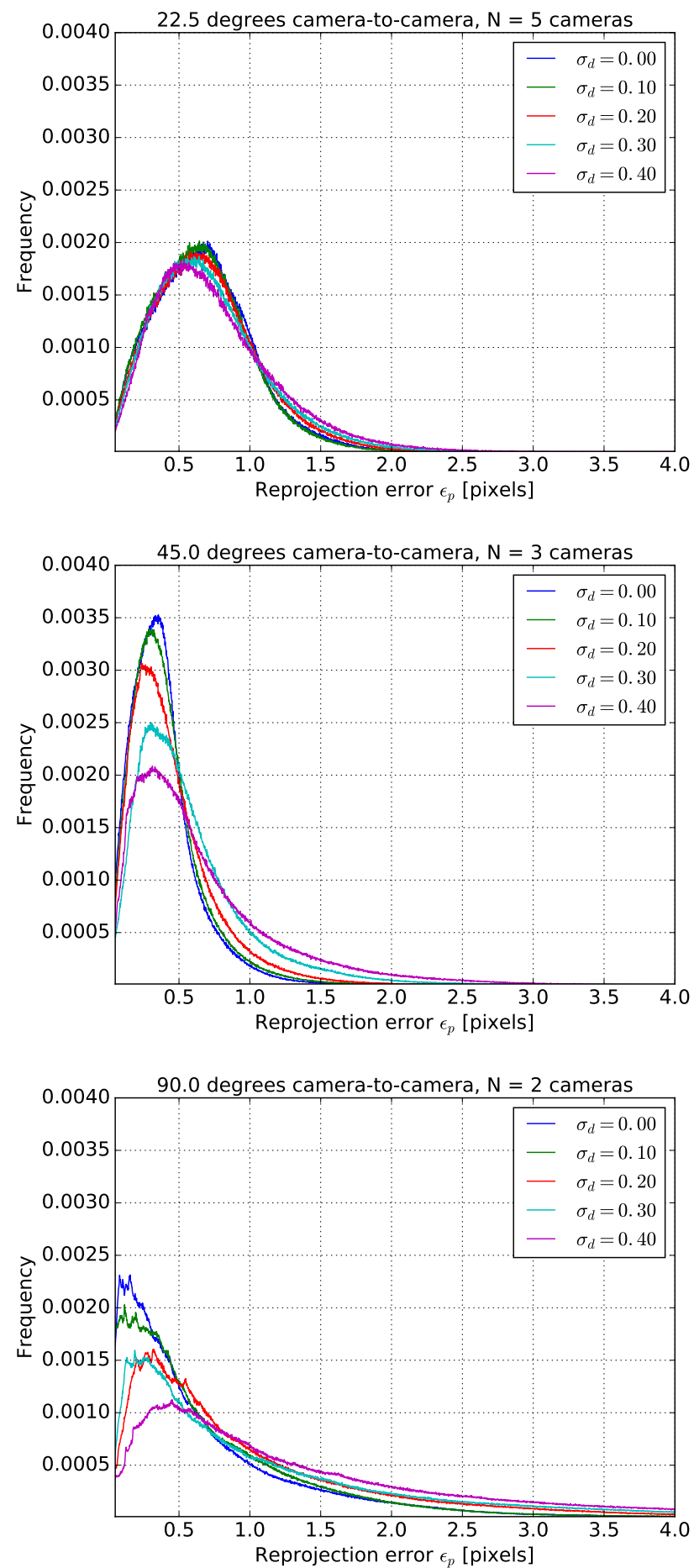

Fig. 6. Distribution of reprojection errors with varying noise magnitude $\sigma_{d}$, computed for the relative pose between outermost cameras at 90 degrees, with $N-2$ cameras in between. 
Table 1. Comparison of rotation errors.

\begin{tabular}{lr}
\hline Method & Rotation error \\
\hline Ours & $0.04^{\circ}$ \\
Esquivel et al. [6] & $0.5^{\circ}$ \\
Antone et al. [2] & $0.1^{\circ}$ \\
Agrawal et al.[1] & $1^{\circ}$
\end{tabular}

The accuracy of the calibration in terms of the absolute pose error is good and is likely to exceed the precision of tools like tape measures and protractors. However, the reprojection error distributions in figure 6 indicate that the calibration error is not negligible but should be accounted for in a structure-from-motion system. In particular, while the error mode is relatively moderate, the heavy tail of the two-camera calibration setup is remarkable and motivates the use of additional cameras during calibration, even for limited-overlap configurations.

The calibration code and calibration quality estimation toolbox, along with the dataset and evaluation code, is available on GitHub ${ }^{1}$.

\section{Conclusion}

We have presented a simple and novel extrinsic calibration method and an evaluation framework for determining the expected quality using synthetic data. We have shown that the system achieves good results with a predictable impact on the intended application in terms of reprojection error distributions. The method is based on off-the-shelf components and is intended for multi-camera setups with small or no overlapping fields-of-view. The procedure is robust and simple to implement which makes it especially suited for industrial applications. Acknowledgement: This work was funded in part by Vinnova, Sweden's innovation agency and Daimler AG.

\section{References}

1. Agrawal, A.: Extrinsic camera calibration without a direct view using spherical mirror. In: Proceedings of the IEEE International Conference on Computer Vision. pp. 2368-2375 (2013)

2. Antone, M., Teller, S.: Scalable extrinsic calibration of omni-directional image networks. International Journal of Computer Vision 49(2), 143-174 (2002)

3. Bradski, G., et al.: The opencv library. Doctor Dobbs Journal 25(11), 120-126 (2000)

4. Carrera, G., Angeli, A., Davison, A.J.: Slam-based automatic extrinsic calibration of a multi-camera rig. In: Robotics and Automation (ICRA), 2011 IEEE International Conference on. pp. 2652-2659. IEEE (2011)

\footnotetext{
${ }^{1}$ https://github.com/midjji/non-overlapping-extrinsic-cameracalibration.git
} 
5. Dong, S., Shao, X., Kang, X., Yang, F., He, X.: Extrinsic calibration of a nonoverlapping camera network based on close-range photogrammetry. Applied Optics 55(23), 6363-6370 (2016)

6. Esquivel, S., Woelk, F., Koch, R.: Calibration of a multi-camera rig from nonoverlapping views. In: Joint Pattern Recognition Symposium. pp. 82-91. Springer (2007)

7. Foundation, B.: Blender version 2.78c (2017), https://www.blender.org/

8. Hedborg, J., Forssén, P.E., Felsberg, M.: Fast and accurate structure and motion estimation. Advances in Visual Computing pp. 211-222 (2009)

9. Hesch, J., Mourikis, A., Roumeliotis, S.: Extrinsic camera calibration using multiple reflections. Computer Vision-ECCV 2010 pp. 311-325 (2010)

10. Kumar, R.K., Ilie, A., Frahm, J.M., Pollefeys, M.: Simple calibration of nonoverlapping cameras with a mirror. In: Computer Vision and Pattern Recognition, 2008. CVPR 2008. IEEE Conference on. pp. 1-7. IEEE (2008)

11. Lébraly, P., Deymier, C., Ait-Aider, O., Royer, E., Dhome, M.: Flexible extrinsic calibration of non-overlapping cameras using a planar mirror: Application to vision-based robotics. In: Intelligent Robots and Systems (IROS), 2010 IEEE/RSJ International Conference on. pp. 5640-5647. IEEE (2010)

12. Liu, Q., Sun, J., Liu, Z., Zhang, G.: Global calibration method of multi-sensor vision system using skew laser lines. Chinese Journal of Mechanical Engineering 25(2), 405-410 (2012)

13. Liu, Z., Zhang, G., Wei, Z., Sun, J.: Novel calibration method for non-overlapping multiple vision sensors based on 1d target. Optics and Lasers in Engineering 49(4), 570-577 (2011)

14. Long, G., Kneip, L., Li, X., Zhang, X., Yu, Q.: Simplified mirror-based camera pose computation via rotation averaging. In: Proceedings of the IEEE Conference on Computer Vision and Pattern Recognition. pp. 1247-1255 (2015)

15. Maddern, W.: Industry solutions: Autonomous vehicle driven by vision. Vision Systems Design 22(2) (2 2017), http://www.visionsystems.com/articles/print/volume-22/issue-2/features/industry-solutionsautonomous-vehicle-driven-by-vision.html

16. Pagel, F.: Extrinsic self-calibration of multiple cameras with non-overlapping views in vehicles. In: IS\&T/SPIE Electronic Imaging. pp. 902606-902606. International Society for Optics and Photonics (2014)

17. Rodrigues, R., Barreto, J., Nunes, U.: Camera pose estimation using images of planar mirror reflections. Computer Vision-ECCV 2010 pp. 382-395 (2010)

18. Sturm, P., Bonfort, T.: How to compute the pose of an object without a direct view? In: Asian Conference on Computer Vision. pp. 21-31. Springer (2006)

19. Takahashi, K., Nobuhara, S., Matsuyama, T.: A new mirror-based extrinsic camera calibration using an orthogonality constraint. In: Computer Vision and Pattern Recognition (CVPR), 2012 IEEE Conference on. pp. 1051-1058. IEEE (2012)

20. Zhang, Z.: A flexible new technique for camera calibration. IEEE Transactions on pattern analysis and machine intelligence 22(11), 1330-1334 (2000) 